\title{
Minimally invasive subcutaneous anterior fixation of pelvic fractures in the elderly: case report and literature review
}

\author{
Sven Strydom, ${ }^{1 *(1)}$ Christian H Snyckers ${ }^{2}$ \\ 1 Department of Orthopaedics, University of Pretoria, Kalafong Tertiary Hospital, Pretoria, South Africa \\ ${ }^{2}$ Private practice, Eugene Marais Hospital, Pretoria, South Africa \\ *Corresponding author: svenstrydom@gmail.com
}

Citation: Strydom S, Snyckers $\mathrm{CH}$. Minimally invasive subcutaneous anterior fixation of pelvic fractures in the elderly: case report and literature review. SA Orthop J 2021;20(4):240-245. http://dx.doi. org/10.17159/2309-8309/2021/ v20n4a8

Editor: Prof. Sithombo Maqungo, University of Cape Town, Cape Town, South Africa

Received: August 2020

Accepted: February 2021

Published: November 2021

Copyright: @ 2021 Strydom S. This is an open-access article distributed under the terms of the Creative Commons Attribution Licence, which permits unrestricted use, distribution and reproduction in any medium, provided the original author and source are credited.

Funding: No funding was received for this study.

Conflict of interest: The authors declare they have no conflicts of interest that are directly or indirectly related to the research.

\begin{abstract}
Background

As our population ages, the incidence of pelvic fragility fractures will rise accordingly. Despite these fractures having similar mortality rates to proximal femur fractures, there exist discrepancies between the management of these injuries. Although a number of pelvic fragility fractures can be treated successfully with conservative means, early treatment with appropriate surgical means should be considered in those failing conservative treatment or with unstable fracture patterns.
\end{abstract}

\section{Case report}

We present an 84-year-old female who sustained a pelvic fragility fracture after a low-energy fall. Despite adequate conservative treatment, she was unable to mobilise. She was taken for anterior and posterior fixation, using our modified minimally invasive subcutaneous technique (the Bridging Infix) for anterior fixation. At the six-week follow-up she had regained full independent mobility. She had three syncope-related falls during this period, but radiographs revealed no sign of implant displacement. One year after her surgery she had complete union of her fracture, good function and no desire to have the implant removed.

\section{Discussion}

With the expected increase in pelvic fragility fractures due to the growing elderly population, our understanding of these injuries has begun to change. Occult posterior ring injuries have been described in up to $80 \%$ of cases, while fracture progression to unstable patterns can occur in up to $15 \%$ of stable patterns. Despite conservative management being the primary treatment of choice, these patients suffer morbidity and mortality rates comparable to proximal femur fractures. Early appropriate surgical management should be considered in patients failing to mobilise. Various surgical techniques have been described, each with their own advantages and disadvantages. Newer minimally invasive techniques are gaining favour, especially for use in elderly patients. These constructs combine the low profile benefits of internal plate fixation with ex-fix principles.

\section{Conclusion}

The Bridging Infix is a modified technique for minimally invasive subcutaneous anterior pelvic fixation. Its use can strongly be considered by even the general orthopaedic surgeon in cases where patients are too frail for extensive or invasive surgeries, such as open reduction and internal fixation with plate and screws.

Level of evidence: Level 5

Keywords: pelvic fracture, anterior pelvic fixation, elderly, minimally invasive

\section{Introduction}

As our population ages, the incidence of traumatic and insufficiency pelvic fractures among the elderly is rising. ${ }^{1}$ This phenomenon highlights the current common misdiagnosis and management of, particularly, the insufficiency pelvic fractures. Isolated pubic rami fractures respond well to conservative management with minimal pain and a quick return to mobility. A similar management approach applied to complex lateral compression-type pelvic fractures is fraught with pain and prolonged immobility leading to increased morbidity and even death. ${ }^{1}$ Increased use of computerised tomography (CT) scans has shown that up to $80 \%$ of presumed isolated pubic rami fractures are complex lateral compression-type pelvic fractures. ${ }^{2}$

Hopf et al. link neck of femur fractures and pelvic insufficiency fractures in the elderly demographic by stating that they share similar mortality rates. ${ }^{3}$ Despite this, a discrepancy remains between their management. Neck of femur fractures enjoy appropriate and aggressive early treatment leading to a marked reduction in mortality, morbidity and complications, whereas insufficiency pelvic fractures remain misdiagnosed and poorly treated.

As with neck of femur fractures, a percentage of patients with 


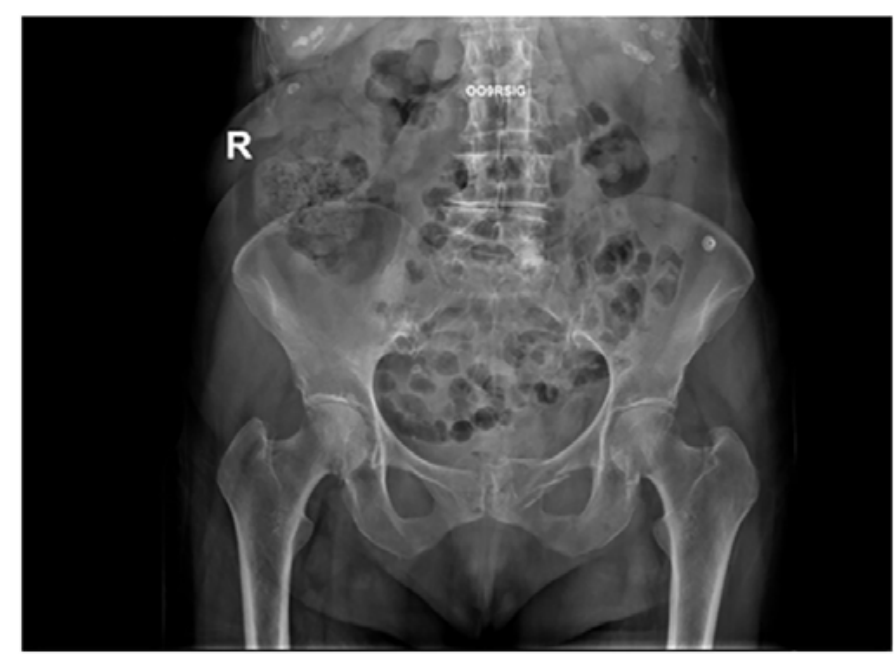

Figure 1. AP radiograph of the pelvis showing left pubic rami fracture, preoperative

stable pelvic fracture patterns can be treated conservatively. Early treatment with appropriate surgery for complex or unstable fractures is, however, essential. Transiliac/sacral screw fixation is an effective treatment for most posterior injuries. At the same time, stabilisations with external fixation, open reduction and internal fixation (ORIF) with sub-muscular plating, retrograde pubic rami screw fixation or newer subcutaneous techniques are possible for anterior instability. ${ }^{1}$ We propose using a novel internal bridge plate and rod technique that combines the extra-pelvic fixation methods of an external fixator with the low-profile advantages of the pelvic bridge and ORIF.

\section{Case report}

We present an 84-year-old female who sustained a pelvic fracture (Figure 1) after a fall while disembarking from a minibus. She was referred to us with a complaint of significant pain and difficulty mobilising despite receiving physiotherapy for the past two days following the injury. Before the injury, the patient was living independently with full mobility and without the use of walking aids. On presentation, the X-rays showed a superior and inferior pubic rami fracture, with the superior rami fracture at the level of the medial edge of the obturator foramen with comminution and extension into the pubis. A CT scan revealed a type $2 B$ fragility fracture of the pelvis comprising anterior pubic rami fractures and a posterior sacral fracture (Figure 2).

She was taken to theatre the following day for fixation (Figure 3) and, under general anaesthesia and a spinal block, a single $6.5 \mathrm{~mm}$ cannulated screw was used to stabilise the posterior arch. A modified minimally invasive subcutaneous technique was used for the anterior arch. Our Bridging Infix technique is a variant of the pelvic bridge described by Hiesterman et al., during which we avoid placing pedicle screws in the pubic tubercle. ${ }^{4}$ Hence the only fixation points are those at the iliac crest, similar to the
INFIX. By avoiding the use of pedicle screws we theoretically reduce the likelihood of patient discomfort caused by prominent screws at the pubis or heterotrophic ossification developing, which was found in around $25-30 \%$ of pedicle screw heads which have been removed. ${ }^{5}$ An additional advantage this construct has over the original pelvic bridge is that it can be used in cases with bilateral pubic rami fractures, as it is not dependent on screw purchase at the pubic tubercle. The patient spent one night in the high care unit for postoperative observations. Physiotherapy was initiated immediately with protected weight-bearing allowed. The patient was able to mobilise safely and was discharged seven days after surgery to a step-down facility for continued physiotherapy.

At the six-week follow-up, the patient was able to walk unaided and pain-free. She had full active range of motion in her hips and was able to perform active straight leg raises bilaterally (Figure 4). She reported having fallen three times since surgery due to syncope and was subsequently referred to a physician for further work-up. She had recovered complete independent mobility but was advised to continue using a walking frame to assist with balance. Radiographs revealed good early union and no signs of displacement, despite her having fallen several times. She was assessed for a final follow-up one year after her surgery. At this time, she reported no discomfort from the Bridging Infix and did not desire the removal of the implant. Radiological examination revealed a good bony union (Figure 5). Her syncope had also improved with appropriate medical therapy.

\section{Discussion}

An estimated $7 \%$ of all osteoporotic fragility fractures affect the pelvic ring; and it has also been shown that $73 \%$ of all pelvic fractures occur in the elderly. ${ }^{6,7}$ A rapidly growing demographic of older adults is evident as life expectancy increases in developed

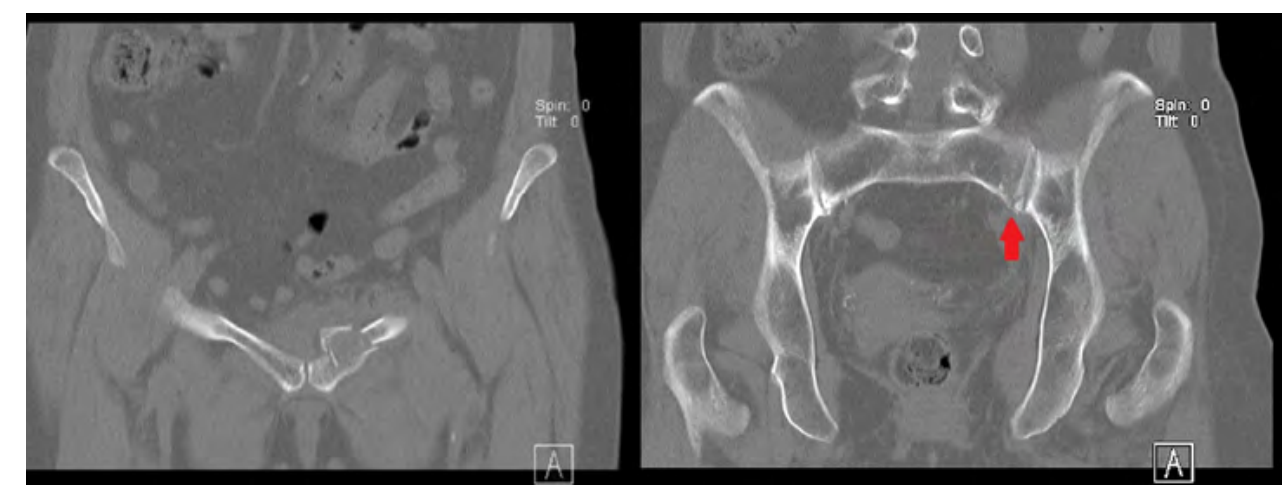

Figure 2. Coronal CT scan views demonstrating pubic ramus and sacral (red arrow) fractures, preoperative

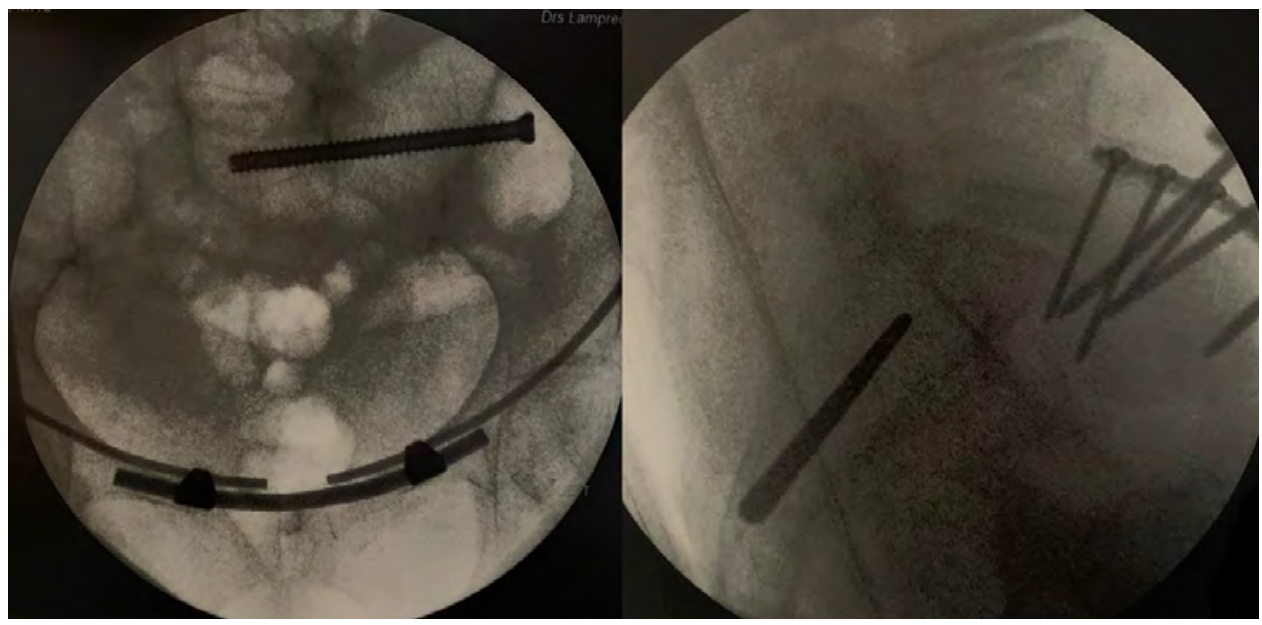

Figure 3. AP and lateral fluoroscopy of pelvis, intraoperative 


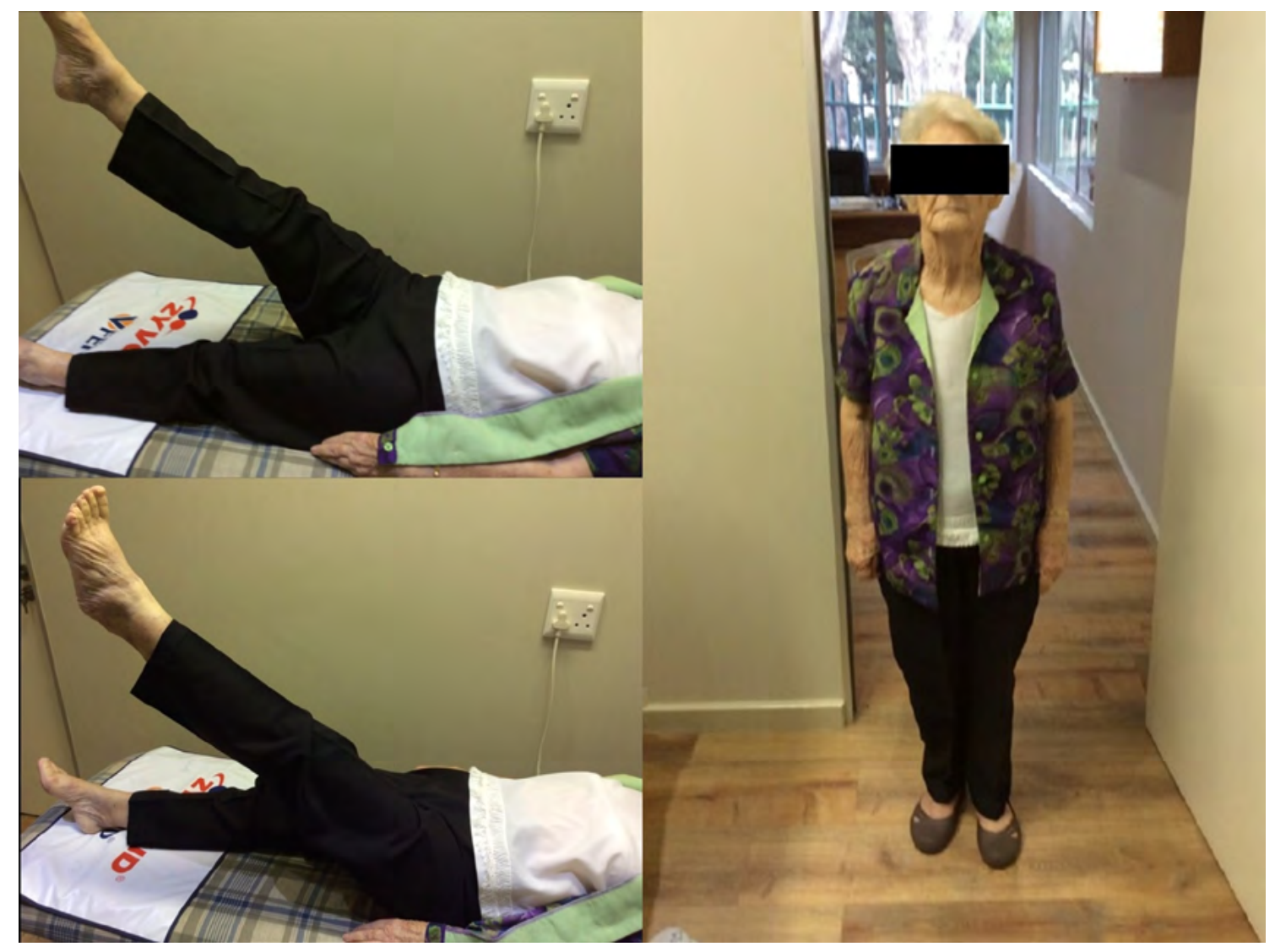

Figure 4. Patient able to perform straight-leg raises (left) and stand independently (right) at six-week follow-up

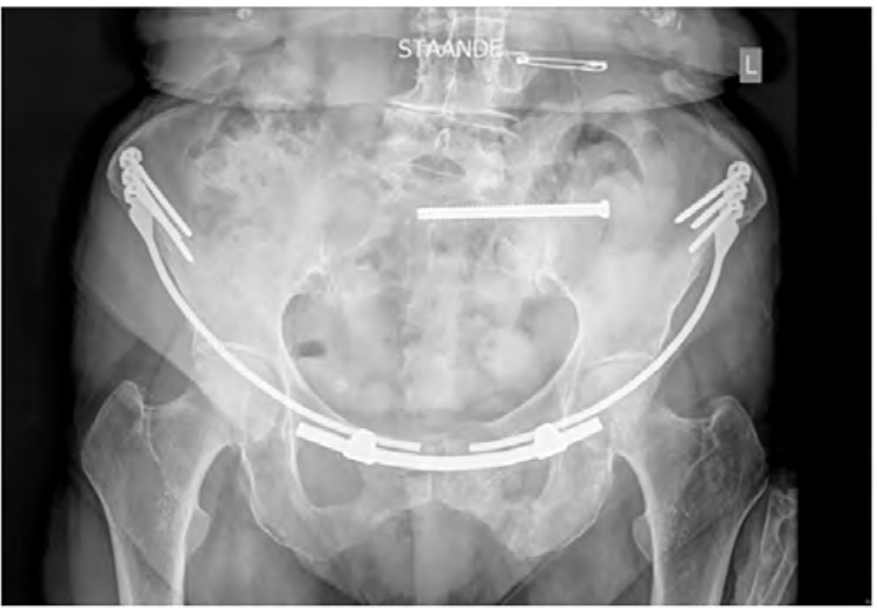

Figure 5. AP radiograph of the pelvis one year after fixation showing good bony union and hardware in situ

countries. The accompanying rise in incidence has made these fractures a common, clinically important problem.1,8,9 The number of pelvic ring fractures among older adults in America increased by $24 \%$ between 1993 and $2010 .^{10}$ Elderly patients are vulnerable due to their age-related physical condition, pre-existing comorbidities, limited rehabilitation capacity, and impaired coping mechanisms. ${ }^{7}$ Pelvic fractures can have a major impact on their quality of life and result in loss of independence..$^{7-9}$ Rehabilitation to independent mobilisation is of critical importance to improve outcomes and prevent common complications such as decubitus ulcers, pneumonia and urinary tract infections, which occur in $58 \%$ of patients treated conservatively. ${ }^{1,7}$ With conservative management as the primary choice of treatment, it is evident that an average hospitalisation duration ranges from 9.3 to 45 days. The time to pain improvement and independent mobilisation varies from four weeks to more than three months, and nearly $50 \%$ of patients report diminished independence afterwards, while only $85 \%$ report complete pain relief after nine months of conservative management. 111 The one-year mortality rate has been reported to reach $27 \%$, while the five-year mortality is as high as $54 \%$, comparable to intertrochanteric and neck of femur fractures among this group. $3,7,11$

Even low-energy trauma such as a fall on the side from a standing position can cause a compression fracture of the lateral mass of the sacrum and associated horizontally running fracture of the superior ramus of the pubic bone. ${ }^{8}$ Differences like this, between young patients with high energy trauma and elderly patients with fragility fractures caused by low energy trauma, combined with the welldocumented change in morphological characteristics of fragility fractures, have led to the proposal of a different classification system for these fractures. ${ }^{8}$ The fragility fracture of the pelvis (FFP) classification is based on increasing degrees of instability. Type I consists of an isolated anterior injury; type II has a non-displaced posterior ring fracture; type III, a displaced unilateral posterior element; and type IV, displaced bilateral posterior ring fractures. Types II to IV may or may not have an associated anterior ring fracture..$^{8,12}$ The literature supports conservative treatment of type I fractures, while type II fractures are best treated with percutaneous techniques to allow early mobilisation. Both types III and IV fractures require more aggressive surgical management due to the associated displacement. ${ }^{12}$ Fracture progression has, however, been described to occur over time, with previously stable patterns progressing to more complex patterns with greater instability leading to the eventual collapse of the pelvic ring. ${ }^{8,9}$ Rommens et al. found progression in nearly $15 \%$ of pelvic fragility fractures that were treated conservatively. ${ }^{9}$ Occult posterior pelvic ring fractures have been described in $54-80 \%$ of elderly patients with pubic rami fractures, $2,13,14$ a statistic highlighting the importance of utilising CT scans to diagnose the fracture pattern correctly. 
Biomechanically, the anterior portion provides $40 \%$ of the pelvic ring's stability, while the posterior elements are responsible for the remaining $60 \% .{ }^{15}$ Traditional methods of fixating the anterior pelvis have included ORIF, external fixation and retrograde pubic rami screws. ${ }^{4,5}$ The most significant benefits of ORIF include allowing for anatomic reduction and there being no need for removal of any implants at a later stage. It remains biomechanically the most rigid construct at the symphysis pubis. ${ }^{16,17}$ Despite the numerous advantages of ORIF, it requires a more extensive surgical approach with a subsequent increase in approach-related complications. ${ }^{6}$ Longer surgeries with greater blood loss not only result in greater physiological stress on the patient's cardiovascular system, but are also associated with a prolonged recovery and increased surgical site infection rate. ${ }^{18}$ Both the Pfannenstiel and modified Stoppa approaches are widely used during anterior pelvic ring ORIF. ${ }^{6}$ With more lateral extension of the incision, the risk of damage to the inguinal canal and its content increases, which can result in ongoing pain symptoms. ${ }^{19}$ It is important to note that there is sparse literature regarding the complications associated with such extensive surgical approaches, specifically in the elderly or frail patient.

External fixation, being a minimally invasive technique, serves to preserve the fracture site's biology and allows for easy removal. ${ }^{5}$ Despite its convenience for the surgeon, it is often unsightly and cumbersome for the patient and has an associated complication rate as high as $62 \% .{ }^{20,21}$ Complications include: pin-tract infection in $2.5-50 \%$ of cases, osteomyelitis, aseptic loosening in up to $19 \%$, patient discomfort, and poor tolerance due to limitations in activities of daily living or mobility or skin impingement, increased difficulty in nursing care and iatrogenic nerve injury. 5,15,20-22 There is also a risk of loss of reduction, which is dependent on the fracture pattern and additional fixation used..$^{20}$ The presence of obesity further increases the risk of loss of reduction and pin-tract complications. ${ }^{5,20}$ Some authors also recommend against external fixation use in fragility fractures. ${ }^{12}$

Another option of anterior fixation is the use of retrograde pubic rami screws. This technique has grown in popularity because it provides a minimally invasive internal fixation option. These screws are not suitable for all fracture types and have a reported loss of reduction in $15 \%$ of cases. ${ }^{23}$ Rommens et al. pointed out that the type of fracture dictates the type of fixation, and Starr et al. clearly showed an increase in instability with fractures medial to the lateral border of the obturator foramen.9,23 They also showed an increase in fixation loss with advanced age and female sex. It is common for all these risk factors to coexist in insufficiency fractures of the pelvis, making retrograde percutaneous screw fixation technically demanding in this subgroup of patients. ${ }^{23}$ This technique also requires a high degree of precision. ${ }^{1}$

The recent development of minimally invasive anterior subcutaneous internal fixation techniques is proving to be a popular alternative means of fracture fixation..$^{5,15,16,21}$ The aim is to limit complications associated with external fixator use while retaining the advantages using internal implants but using the same fixation principles. ${ }^{5,15,20}$ Their application requires minimal soft tissue dissection, which reduces operating time, intraoperative blood loss and length of stay when compared to ORIF.5,21,24 The subcutaneous location also decreases the risk of surgical site infection, eases the burden of nursing care, and avoids interference with rehabilitation and daily activities..$^{5,20,24}$ Biomechanically they provide sufficiently rigid fracture fixation to facilitate bone healing. ${ }^{5}$ Several studies conclusively demonstrated superior stiffness at the pubic symphysis compared to external fixators, while one study by Vigdorchik et al. demonstrated these constructs could provide some indirect compression of the posterior pelvic elements. $5,16,25$ This can prove beneficial when acute stabilisation of the posterior elements is contraindicated. ${ }^{25}$ Long-term follow-up of these techniques show high patient satisfaction and acceptably low complication rates. ${ }^{17,26}$

The first of these novel techniques was originally described in German literature in 2009, but it was Vaidya et al. who described the modified method currently being used, and coined the term INFIX for this technique. ${ }^{5,27}$ Their technique involved the placement of pedicle screws in the supra-acetabular area with a connecting curved rod in the epifascial plane. A potential drawback is the rod which transverses the inferior abdominal wall, thus creating a potential for iatrogenic compression or impingement injuries. Screw placement also requires deep dissection in the interval between the sartorius and tensor fascia latae muscles. ${ }^{5}$

A more recent variation of this minimally invasive technique is the pelvic bridge described in 2012. ${ }^{4}$ This method involves spanning the anterior pelvis between two 'pillars', usually the ipsilateral iliac crest and either the ipsilateral or contralateral pubic symphysis. The bridge is formed with either a low-profile pre-contoured locking plate or a rod-plate construct (originally used for occipito-spinal fusions). ${ }^{4,5}$ The bridge is passed subcutaneously, above the external oblique fascia, along the static anatomic structures namely the iliac crest, inguinal ligament and pubic symphysis. ${ }^{4,5,22}$ The construct design can allow for either selective percutaneous fixation of the compromised hemipelvis without involving the uninjured side, or two separate fixators can be used with an overlap and rod-to-rod connections at the pubic symphysis for bilateral fractures. ${ }^{5}$ These differences in the course of the connecting rod and construct design provide some advantage over the INFIX.

A cadaver study by Reichel et al. compared the INFIX and pelvic bridge techniques. ${ }^{28}$ They noted several challenges with the INFIX application. First, its application was variable and highly dependent on the pedicle screws placement and curvature of the rod, which results in an increased risk of impingement due to the patient's body habitus or when greater flexion is attempted at the waist. Since the connecting rod did not mirror static anatomic structures like the pelvic bridge, there also remained the risk of the rod twisting or being misplaced while securing the locking caps. Lastly the pedicle screw depth is a subjective measure as it lies several centimetres above the bone; placement at the incorrect depth can lead to either patient discomfort from prominent hardware or impingement of underlying structures. The pelvic bridge utilises four points of fixation, which aids in reducing the chance of misapplying the device in a manner that impinges neurovascular structures. Despite the literature showing that supra-acetabular screws are superior to iliac crest screws, there are no biomechanical studies directly comparing the INFIX and pelvic bridge constructs. ${ }^{16,28}$ Theoretically the four-point fixation of the pelvic bridge may impart greater overall biomechanical strength when compared to the twopoint fixation of the INFIX.4,28 Another anatomic study demonstrated that despite variations in pelvic anatomy such as pelvic brim width, the pre-contoured rods or plates did not violate any neurovascular structures. ${ }^{29}$ The pelvic bridge does, however, have a theoretical risk of bladder injury with erroneous drilling and screw placement into the pubic symphysis, but no such case has been described in the literature to date. ${ }^{28}$

Several studies have looked at the complication rates associated with the INFIX, while literature on the pelvic bridge technique is still sparse. One of the most common complications of the INFIX found in the literature is lateral femoral cutaneous nerve (LFCN) neuropraxia, which most studies found in $25-32 \%$ of all cases. 5,15,17,20,21 Two studies found even higher rates of LFCN neuropraxia, occurring in $55-57 \%$ of cases. ${ }^{30,31}$ Patients will present with numbness over the anterolateral thigh; this is, however, well tolerated by most patients and the majority resolve with time after removal of the implant. 5,15,17,21,28 These findings 
are well explained by Reichel et al., who state that despite the INFIX having a significantly greater distance from most anatomic structures when compared to the pelvic bridge, it lacks a 'safety margin' in $90.9 \%$ of specimens between the LFCN and pedicle screws. ${ }^{28}$ In addition, LFCN injury may be caused by difficulty in locating the nerve during dissection to place the pedicle screws. ${ }^{5,28}$ A review by Vaidya et al. could not find a recommendable solution to prevent this complication with INFIX application. ${ }^{17}$ The pelvic bridge was proposed as an alternative to minimise the risk of LFCN neuropraxia, as the implant remains a significantly greater distance from the LFCN when compared to the INFIX. ${ }^{28}$ It must, however, be noted that anatomical variations of the LFCN has been described in 2.9 to $4 \%$ of the population, which can place the nerve at risk when dissecting near the iliac wing to place the pelvic bridge. ${ }^{5}$

Another common complication noted with the INFIX is heterotrophic ossification around the pedicle screws, which occurs in $21-36 \%$ of cases. $., 15,17,20,21$ Despite being asymptomatic, some authors do recommend the use of preventative measures such as prophylactic use of non-steroidal anti-inflammatory drugs or radiotherapy. 15,17,20 Other complications occur more rarely and include a surgical site infection rate of $3 \%$, but no documented cases progressed to osteomyelitis. ${ }^{17}$ Acute and delayed onset femoral nerve neuropraxia occurred in $1 \%$ due to seating the rod too deep in overweight patients. ${ }^{29}$ These injuries were more likely to be permanent despite implant removal compared to LFCN injuries, with one study showing total recovery in only one out of eight patients. ${ }^{32}$ Vascular occlusion occurred in one case, but was diagnosed early enough to prevent long-term complications. ${ }^{33}$ Aseptic loosening with loss of reduction and entrapment of the anterior abdominal wall causing severe discomfort has also been described. ${ }^{5}$ Vaidya et al. found up to $7.3 \%$ of INFIXs required early revision due to complications. ${ }^{17}$ Fortunately, the majority of complications were considered minor; however, the potential for devastating complications does exist and this underscores the importance of education on the use and improvement of techniques to decrease the associated risks. ${ }^{5}$

As mentioned, there are limited studies regarding the complications encountered with the pelvic bridge. Cole et al. found the overall complication rate with the pelvic bridge technique to be $4 \% .^{5}$ The complications they encountered included superficial wound infection, one asymptomatic pubic ramus non-union and temporary LCFN neuropraxia. ${ }^{5,33}$ In another study they also demonstrated significantly less pain and discomfort at follow-up when compared to an external fixator. ${ }^{33}$

A common disadvantage for both minimally invasive techniques is that hardware needs to be removed in theatre at a later stage. This is recommended as there are no long-term studies available, and potentially deleterious consequences from leaving the device in situ are unknown.5,22 Pressure over the device can also cause unnecessary discomfort for the patient. ${ }^{22}$ Current literature recommends removal of the pelvic bridge between eight and 16 weeks, before excessive soft tissue ingrowth can occur. ${ }^{5,34}$ Campbell et al. described a novel endoscopic technique utilising incisions smaller than those of the original procedure, to successfully remove the implant with excellent preliminary results. ${ }^{34}$ They also noted that the more expensive rod-plate construct was easier and less traumatic to remove compared to the locking-plate construct. ${ }^{34}$ Since the fracture site is not exposed, there is a risk of soft tissue interposition with resultant non-union, and fracture fragments that may cause neural or organ compression cannot be removed. ${ }^{24}$

Being novel techniques, the ideal indications and contraindications of the INFIX and pelvic bridge are still being defined.
Current indications include both high and low energy pelvic ring injuries requiring anterior stabilisation, in either isolated anterior injuries or after posterior stabilisation has been achieved. It is also proving to be advantageous in coagulopathic patients to minimise intraoperative blood loss, in patients expected to have a protracted ICU course to facilitate nursing care and decrease infection risk, and in those requiring the prone position later for procedures such as spinal surgery. ${ }^{5,22,24}$ Patients with morbid obesity also tolerate subcutaneous devices better than an external fixator; the pelvic bridge has been suggested to be superior to the INFIX regarding its ease of application as the anatomic landmarks are identified more easily in obese patients. ${ }^{5,22,24}$

Contraindications include pure ligamentous dislocation at the pubic symphysis or iliac wing dislocations; severe degloving soft tissue injuries or active infection in the suprapubic or iliac crest areas; open pelvic fractures with peritoneal contamination; pregnancy; and haemodynamically unstable pelvic ring injuries requiring rapid stabilisation for life-saving reasons. ${ }^{5,24}$ It has been noted that the pelvic bridge should never be used as the sole fixation method in combined anterior and posterior instability. ${ }^{24}$ Stable pelvic fractures in patients who are able to mobilise effectively with partial weightbearing and acetabular fractures involving the anterior column have also been suggested as potential contraindications. ${ }^{24}$

A suggested postoperative rehabilitation programme consists of weight-bearing as tolerated in the first six weeks, followed by full weight-bearing. During this time there is no restriction on hip range of motion. ${ }^{4,22}$

It is also proving to be an appealing surgical option in the elderly with osteoporotic bone to provide pain relief and allow rapid mobilisation while minimising surgical risk. ${ }^{5,22}$ The use of bone cement can further augment screw purchase in osteoporotic bone. ${ }^{5,9}$ Taking into consideration that prolonged immobilisation is associated with potentially fatal complications in the elderly, early surgical treatment and mobilisation has been suggested to improve mortality rates. ${ }^{5,6,9}$ This was confirmed by Osterhoff et al. who found that surgically treated patients who survived the initial two years post injury had a better long-term survival compared to conservatively treated patients. ${ }^{35}$ Their slightly higher mortality rate in the first two years may be explained by the perioperative risks inherent with anaesthesia and surgery in the elderly. They thus recommend considering early surgical management for patients with a greater than two-year life expectancy. In addition to the improved long-term survival, surgical fixation is gaining favour due to its better pain relief, faster mobilisation and shorter recovery period compared to conservative management. ${ }^{3}$

\section{Conclusion}

The incidence of pelvic fragility fractures is on the rise as our population ages. Underestimating the seriousness of these fractures and inappropriate identification and management thereof can lead to significant morbidity and mortality. This is comparable to conservative management of proximal neck of femur fractures. This case demonstrates the effectiveness of appropriate, aggressive surgical intervention in complex fragility pelvic fracture. Here the use of minimally invasive subcutaneous anterior fixation and percutaneous posterior stabilisation provides for rapid pain relief and allows early mobilisation. As the literature demonstrates, our understanding of pelvic fragility fractures is expanding and with that the place and value of early, aggressive and appropriate surgical fixation is becoming more evident.

\section{Ethics statement}

Written consent was received to use photos, radiographs and clinical data from the patient and is available on request. 


\section{Declaration}

The authors declare authorship of this article and that they have followed sound scientific research practice. This research is original and does not transgress plagiarism policies.

\section{Author contributions}

SS: Contributed to the conceptualisation, data collection and writing of the article CHS: Primary treating surgeon of the case presented, contributed to the conceptualisation, data collection and writing of the article

\section{ORCID}

Strydom S (D) https://orcid.org/0000-0002-6288-4388

Snyckers CH https://orcid.org/0000-0002-6297-894X

\section{References}

1. Oberkircher L, Ruchholtz S, Rommens P, et al. Osteoporotic pelvic fractures. Dtsch Arztebl Int. 2018;115(5):70-80. https://doi.org/10.3238/arztebl.2018.0070.

2. Tosounidis G, Wirbel R, Culemann U, Pohlemann T. Fehleinschätzung bei vordere beckenringfraktur im höheren lebensalter [Misinterpretation of anterior pelvic ring fractures in the elderly]. Unfallchirurg. 2006;109(8):678-80. https://doi.org/10.1007/s00113-006-1098-1.

3. Hopf J, Krieglstein C, Müller L, Koslowsky T. Percutaneous iliosacral screw fixation after osteoporotic posterior ring fractures of the pelvis reduces pain significantly in elderly patients. Injury. 2015;46(8):1631-36. https://doi.org/10.1016/j.injury.2015.04.036

4. Hiesterman T, Hill B, Cole P. Surgical technique: A percutaneous method of subcutaneous fixation for the anterior pelvic ring: The pelvic bridge. Clin Orthop Relat Res. 2012;470(8):2116-23. https://doi.org/10.1007/s11999-012-2341-4.

5. Cole $P$, Dyskin E, Gilbertson J. Minimally invasive fixation for anterior pelvic ring disruptions. Injury. 2015;46(S3):S27-34. https://doi.org/10.1016/S0020-1383(15)30008-5.

6. Küper M, Trulson A, Stuby F, Stöckle U. Pelvic ring fractures in the elderly. EFORT Open Rev. 2019;4(6):313-20. https://doi.org/10.1302/2058-5241.4.180062.

7. Banierink $H$, Ten Duis $K$, De Vries $R$, et al. Pelvic ring injury in the elderly: fragile patients with substantial mortality rates and long-term physical impairment. PLoS One. 2019;14(5):e0216809. https://doi.org/10.1371/journal.pone.0216809.

8. Rommens P, Wagner D, Hofmann A. Fragility fractures of the pelvis. JBJS Rev 2017;5(3):e3. https://doi.org/10.2106/JBJS.RVW.16.00057

9. Rommens $\mathrm{P}$, Arand $\mathrm{C}$, Hopf $\mathrm{J}$, et al. Progress of instability in fragility fractures of the pelvis: An observational study. Injury. 2019;50(11):1966-73. https://doi.org/10.1016/j. injury.2019.08.038.

10. Sullivan MP, Baldwin KD, Donegan DJ, et al. Geriatric fractures about the hip: divergent patterns in the proximal femur, acetabulum, and pelvis. Orthopedics. 2014;37:151-57. https://doi.org/10.3928/01477447-20140225-50.

11. Maier G, Kolbow K, Lazovic D, et al. Risk factors for pelvic insufficiency fractures and outcome after conservative therapy. Arch Gerontol Geriat. 2016;67:80-85. https://doi. org/10.1016/j.archger.2016.06.020.

12. Rommens $P$, Ossendorf $C$, Pairon $P$, et al. Clinical pathways for fragility fractures of the pelvic ring: personal experience and review of the literature. J Orthop Sci. 2015;20(1):1-11 https://doi.org/10.1007/s00776-014-0653-9.

13. Lau $T$, Leung $F$. Occult posterior pelvic ring fractures in elderly patients with osteoporotic pubic rami fractures. J Orthop Surg (Hong Kong). 2010;18(2):153-57. https://doi. org/10.1177/230949901001800205

14. Alnaib $\mathrm{M}$, Waters $\mathrm{S}$, Shanshal $\mathrm{Y}$, et al. Combined pubic rami and sacral osteoporotic fractures: a prospective study. J Orthop Traumatol. 2012;13(2):97-103. https://doi. org/10.1007/s10195-012-0182-2.

15. Yin $Y$, Luo J, Zhang R, et al. Anterior subcutaneous internal fixator (INFIX) versus plate fixation for pelvic anterior ring fracture. Sci Rep. 2019:9(1):2578. https://doi.org/10.1038/ s41598-019-39068-7.

16. Vigdorchik J, Esquivel A, Jin X, et al. Biomechanical stability of a supra-acetabular pedicle screw internal fixation device (INFIX) vs external fixation and plates for vertically unstable pelvic fractures. J Orthop Surg Res. 2012;7(1):31. https://doi.org/10.1186/1749-799X-7-31.

17. Vaidya R, Woodbury D, Nasr K. Anterior subcutaneous internal pelvic fixation/INFIX A systematic review. J Orthop Trauma. 2018;32(S6):S24-30. https://doi.org/10.1097/ BOT.0000000000001248.

18. Rommens $P$, Wagner $D$, Hofmann $A$. Minimal invasive surgical treatment of fragility fractures of the pelvis. Chirurgia. 2017;112(5):524-37. https://doi.org/10.21614/chirurgia.112.5.524.

19. Grewal I, Starr A. What's new in percutaneous pelvis fracture surgery? Orthop Clin North Am. 2020;51(3):317-24. https://doi.org/10.1016/j.ocl.2020.02.010.

20. Vaidya $R$, Kubiak $E$, Bergin $P$, et al. Complications of anterior subcutaneous internal fixation for unstable pelvis fractures: A multicenter study. Clin Orthop Relat Res. 2012;470(8):2124 31. https://doi.org/10.1007/s11999-011-2233-z.

21. Steer R, Balendra G, Matthews J, et al. The use of anterior subcutaneous internal fixation (INFIX) for treatment of pelvic ring injuries in major trauma patients, complications and outcomes. SICOT J. 2019;5:22. https://doi.org/10.1051/sicotj/2019019.

22. Cole P, Hesse D, Dugarte A, Dyskin E. The pelvic bridge. JBJS Essent Surg Tech 2017;7(3):e20. https://doi.org/10.2106/JBJS.ST.15.00085.
23. Starr A, Nakatani T, Reinert C, Cederberg K. Superior pubic ramus fractures fixed with percutaneous screws: What predicts fixation failure? J Orthop Trauma. 2008;22(2):81-87. https://doi.org/10.1097/BOT.0b013e318162ab6e.

24. Scheyerer M, Zimmermann S, Osterhoff G, et al. Anterior subcutaneous internal fixation for treatment of unstable pelvic fractures. BMC Res Notes. 2014;7:133. https://doi. org/10.1186/1756-0500-7-133.

25. Vigdorchik J, Esquivel A, Jin X, et al. Anterior internal fixator versus a femoral distractor and external fixation for sacroiliac joint compression and single stance gait testing: a mechanical study in synthetic bone. Int Orthop. 2013;37(7):1341-46. https://doi.org/10.1007/ s00264-013-1890-9.

26. Müller F, Stosiek W, Zellner M, et al. The anterior subcutaneous internal fixator (ASIF) for unstable pelvic ring fractures. Clinical and radiological mid-term results. Int Orthop. 2013;37(11):2239-45. https://doi.org/10.1007/s00264-013-2032-0.

27. Vaidya $R$, Colen $R$, Vigdorchik J, et al. Treatment of unstable pelvic ring injuries with an internal anterior fixator and posterior fixation: Initial clinical series. J Orthop Trauma. 2012;26(1):1-8. https://doi.org/10.1097/BOT.0b013e318233b8a7.

28. Reichel L, MacCormick L, Dugarte A, et al. Minimally invasive anterior pelvic internal fixation: An anatomic study comparing pelvic bridge to INFIX. Injury. 2018;49(2):309-14. https://doi. org/10.1016/j.injury.2017.12.009.

29. Moazzam C, Heddings A, Moodie P, Cole P. Anterior pelvic subcutaneous internal fixato application: An anatomic study. J Orthop Trauma. 2012;26(5):263-68. https://doi.org/10.1097| BOT.0b013e31823e6b82.

30. Dahill M, McArthur J, Roberts $\mathrm{G}$, et al. The use of an anterior pelvic internal fixator to treat disruptions of the anterior pelvic ring. Bone Joint J. 2017;99-B(9):1232-36. https://doi. org/10.1302/0301-620X.99B9.BJJ-2016-1025.R2.

31. Hoskins W, Bucknill A, Wong J, et al. A prospective case series for a minimally invasive internal fixation device for anterior pelvic ring fractures. J Orthop Surg Res. 2016;11(1):135 https://doi.org/10.1186/s13018-016-0468-9.

32. Hesse $D$, Kandmir $U$, Solberg $B$, et al. Femoral nerve palsy after pelvic fracture treated with INFIX: A case series. J Orthop Trauma. 2015;29(3):138-43. https://doi.org/10.1097/ BOT.0000000000000193.

33. Cole $\mathrm{P}$, Gauger $\mathrm{E}$, Anavian J, et al. Anterior pelvic external fixator versus subcutaneous internal fixator in the treatment of anterior ring pelvic fractures. J Orthop Trauma. 2012;26(5):269-77. https://doi.org/10.1097/BOT.0b013e3182410577.

34. Campbell A, Dugarte A, Ly T. Minimally invasive endoscopic-assisted technique for implant removal after pelvic bridge plating. J Orthop Trauma. 2018;32(11):e457-61. https://doi. org/10.1097/BOT.0000000000001267.

35. Osterhoff G, Noser J, Held U, et al. Early operative versus nonoperative treatment of fragility fractures of the pelvis. J Orthop Trauma. 2019;33(11):e410-15. https://doi.org/10.1097/ BOT.0000000000001584. 